2 过深，易使种子在刚解冻时師吸水萌动，回冻后及 遭冻害。根据各地資料，播种深度，以4-6堰米为 宜。香日德农場的播梁試驗結果，5.12堰米的发芽 率达到了 $97.7 \%$ 。

\section{三、早惷及时耙地松土，破除板結}

播种以后，由于降雪或降雨等原因，会使土壤 造成板結，降低出苗率。因此，冬春降雪以后，应 当及时組織力量, 分散低处过多的积雪; 早春还应 及时进行耙地松士的工作, 以破除板結, 增进出苗 率。

只要䚿点地执行这些实践中已經行之有效的技 术措施，春麦冬播的早熟、高产的效果，一定能够 实現，而当前生产中存在的比較严重的缺苗現象，
也一定能够克服。

当然选择抗寒力較高的小麦品种作为播种材 料，采用条播方法，也都是春麦冬播中不应忽視的 环节。

春麦冬播問題，早在百年以前，甘肃张掖地区 的基众就已采用过。但是在解放以前，这一經驗始 終沒有被总結和研究过。解放以后，特別是大跃进 以来的实践証明，这是一項具有重要意义的革命性 的措施。它不仅对当前的农业生产有着重大的意义; 抹且也为农业科学提出了新的启示。在各行各业大 力支援农业的今天，科学工作者应当对这一問題給 予足够的注意, 进行更深入的总結和呼究, 使其发 揮作用，为大办农业、大办粮食立功。

\title{
人造䊹維和合成䊹維研究 及生产发展的近况
}

刘克 静

近年来，高速度发展的工业，要求提供各种优 异特性的維及其他合成材料，从而促使人造秆維 和合成維的科学研究工作也获得了很快的进 展。 以天然維和从天然䊹維制得的人造䊹維的呼究工 作来颜，有着較久的历史，已經积累了較多的經 驗，目前在工业上的应用也較广泛。但是由于它們 在超分子結构上的特点及大分子之間还存在数量較 多的雨鍵，因而使䊹維大分子常呈較高的显 硬性, 同时, 也由于本身化学結构的局限性, 使它的产品 在弹性、㳔褶緭及耐磨性能方面都比較差，而且在 染色活性、对光和热的稳定性、抗化学䓎剂性能及 抗腐創性能上也存在一些缺点。因此，在不同程度 上限制了它的产品在各种工业上应用的范围。为了 徼性能更为优异的新型人造維，継續提高品种 貭量和进一步深入探索它的制备过程的基本规律以 改进生产技术，近年来曾进行了大量的科学哳究工 作。

合成䊹維具有强度高、耐灀、弹性好、抗腐蝕
性能較好等优良特点。最近, 随着化学工业的飞速 发展及技术条件的日篮成熟，利用各种資源，如石 油工业副产品、天然气以及农副产品等，来制备各 种合成維的可能性也日盆扩大; 同时，由于工业 及科学研究对合成維的性能,不断提出新的要求, 因此，在短时期內，它有了很大的发展。但由于目 前一些典型的合成糿維在結构性能上还存在一些弱

表 1 全世界人浩釈維与合成釈維近年生产情况 （单位：干吨）

\begin{tabular}{|c|c|c|}
\hline 年 份 & 人造納維 & 合成籸維 \\
\hline 1958 & 2,363 & - \\
\hline 1959 & 2,500 & 575 \\
\hline
\end{tabular}

点 (如热稳定性低、形变大、織物透气性差等), 以 及受到生产技术复杂、建厂不易、成本高等因素的 限制，侣不能在紡織工业方面超过人造䊹維的地位 或取而代之。因此研究解决这些問題和継繻探索新 
型合成秄維就具有同等重要的意义。

\section{人造紟維和合成納維工业发展的現状}

人造糿維方面:随着工业生产的日益扩大,广泛 利用非針叶树糿維素资源作为人造維的原料，已 經在不同的生产規模上成功地得到了应用，例如闊 叶树紙浆(如小毛样、樺木等)及非木村資源（如芦 䒨、甘蓔渣、竹类等)都已被用来制作人造糿維。在 工艺过程方面，一些简化及連績化的方法已在一定 范围內代替了原有的經典方法，如用直接法制备粘 液絲，以及粘液的連綂化紡絲和后处理等。在改善 产品性能及降低原材料和䓎品的消耗等方面，也都 陆䜌在生产規模上取得了不少成就。随着研究工作 的継繶深化与开展，具有特殊性能的新型人造糿維 不断付諸生产，一些具有低潤胀性，耐褶緆、而洗桬 而干湿强度均較高的粘液人造种維, 如商品 Covar, Topab, Avisco-YL 型納維(具有干强度 4.4克/倠，湿 强度 3.4 克/袋，可能是粘液秄䧴經一定类型化合物 如甲醀、乙二醛、对垟甲基腿等处理后，使其大分 子間存在少量交联而制得)，已得到广泛的应用，如 制作日常衣着及地秋等等。

在醋酸秆䊒工业中，現在已桱可以明确地看出 这一点，由三醋酸酯所制得的糿維，在制备过程和

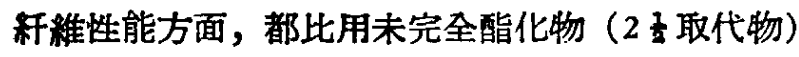
制得的䊹維要优越。一种具有較高保溫能力的醋酸 䊹維(Typ K 及 Hc) 已經投入生产。由汽相或液相局 部乙酸化天然䊹維素(棉、麻)所得的产品，具有較 高的耐溫性能和抗菌性能，伸度及弹性也較佳。現 在, 通过广泛的化学变性途径以改善糿維素糿維的 机栈性能方面，已具生产规模的有：染料活性及离 子交換活性都比較高的羥乙基化、局部䍪甲基化及 胺处理的糿維素秆維，抗細菌能力、热稳定性及耐

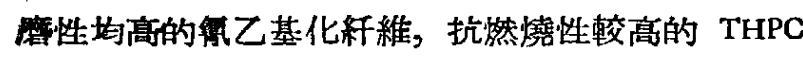
秆維及 BAP、APO 糿維(糿維素与四垟甲基氮化磷及 三丙烯磷酯、三叮嗪磷醉分別作用)，以及 THPC 及 APO 混合处理的糿維等。

合成秆維方面：由一些单聚体和共聚体制得的 具有各种特性的合成維，近年来也不断地从研究 試驗阶段过渡到生产阶段。現将几种典型产品的情 况分別簡迅如下:
聚酸胺类: 爭取生产过程連縟化，改进技术， 稳定抹提高盾量，降低成本，几乎是对一些現已生 产的各种类型聚酸胺維的共同要求，对那些能被 用来制作日常衣着的糿維而言，更是如此。一些具 有高强度及抗裂构(老化)性、抗化学获剂性的聚醚 胺与含氟聚合物的共聚秆維（如 Ftorlon），已可小 量生产。由己二异氧酸及丁二醇聚合制得的强度較 高的聚氨基甲酸糿維，現在也已生产。

聚丙烯腈类: 具有类似羊毛性能的聚丙烯腈䊹 維，早已在各国市場上出現。但目前在工业上仍对 其聚合方式(分散聚合、乳液聚合、溶液聚合等)、 不同紡絲方法及工艺过程連繢化进行着 不断的改 进。而一些具有染色活性及溶解性能好、弹性、酎磨 摖性、抗化学药剂性和热稳定性更高的新型共聚䊹 維，目前地在逐漸增多抹投入生产。其中主要的品 种有:（1）与乙烯醋酸酯共聚的糿維, 如商品 Acrilan 等;（2)与乙烯吡啶共聚的糿維; (3) 与氮乙烯类共 聚的䊹維，如商品 Zefran, Dyneb, Ssaniw; (4)与丙 烯酰胺及丙烯酸酯类共聚的維，如 Verel, Creslan （或称 X 51 及 X 54 秆維）等。

家乙烯类秄䊒: 目前已制得了不少能用于日常 农着的商品納維，但由于它們的热稳定性低，因而 适用范围受到一定限制。但商品 Zefran 等共聚䊹 維，其热稳定性則已有显著提高。

聚酯类：目前，由对苯二甲酸与二醇制得强度 高而弹性优良的、类似羊毛的聚酯䊹維，在市場上 的供应量已逐年增大。但何需改进它的生产技术条 件，提高它的染色活性和热稳定性扑降低成本。通 过一些共聚糿維以改善它的染色活性的方法, 現已 在生产规模上进行試䲆。

其他如由聚乙烯醇及其衍生物制得的糿維，也 有相当大的生产规模。小量的聚丙烯糿維已在国外 市場上出現。不过它的染色活性較差，易受热及氧 化影响而引起裂构。在成本上虽然它可能是合成糿 維中最低的一种，但只有在上述問題得到解决 后， 才有可能大量发展。作为工业用的聚苯乙烯及四楀 乙烯䊹維(Teflon), 目前也有小量生产。惟后者在紡 絲技术上还有較大困难，目前采用的是喷雾紡䋛 法，垖将聚四氟乙稀研成极細的粉末，与高沸点的 碳氫化合物混合，再从喷稀头中挤压出絲。 
最近在紡織工业上出現了一个全新的而发展地 較快的趋势，咱利用棉、人造䊹維及合成紒維，經 适当加工后制成无紡織物。在这方面，継續扩大使 用一些新型紒維和粘合剂，改进工艺过程及加工設 备等, 是必須予以研究解决的問題。

\section{人造秆維和合成秆維研究的发展情况}

工业生产的发展促使科学研究不断前进。在寻 戟新型聚合物，研究天然高聚物的反应方式及聚合 反应的基本規律，探討納維結构及物化性能的关系 等方面, 研究成果正日谷丰富。但从近年来的研究 秥果来看，发展是不平衡的。例如，与直接阔明糿 㱷結构及其性能有关的高分子物理方面的研 究工 作，还是在最近阶段才逐漸发展起来。目前有关的 研究結果大部分还停留在經驗地宏观地說明問 題, 至于进一步系統地探索其本稹联系，总結規律，还 需有关的呼究工作者継績努力。

秆維的物理机械性能，不仅与大分子基本单元 的結构有关，而且与它的超分子結构（包括超分子 序态結构及形态結构)，分子的大小和长度，分子 运动方式和官能团的数量与分布等都有 联 系; 同 时，它与位于晶区及无定形区* 內的大分子間的相 互位圈及交联情况、取向度及結晶度等与物理性能 的关系也极溜切。但由于測定方法的局限，这些問 題至今还不明确。最近利用 $\mathrm{X}$ 射綫小角閃射、电子 衍射图譜及紅外光譜等方法来綜合地研 究結 构特 点，才使上逃問題的解决更趋于合理。

同样在共聚䊹維上問題更为严重，尽管为数繁 多的新型共聚物不断制得，但对共聚体結构状态的 湘定，至今还没有一个滿意的方法。近年来，除了 通过化学裂构、熔化溫度曲綫及溶解度关系等方法 来谽明一部分問題外，也可用热机械曲綫来研究共 聚物結构状态及分子量的分布等問題，或者根据其 静态变化想律(应力应变曲綫及蠕变等)，以进一步 明它的結构变化与机械性能之閒的关系。

无論人造糿維还是合成糿維，往往都是随制备 过程，特別是随成型讨程中工艺条件的不同，而直 接影响到䊹維結构和它的机械性能。如熔螎紡絲过

*目前这些既念还未趋于統一，也还汥有一个完全的 概念,为鿁明方便起見,暫沿用旧名。
程中溫度变化及拉伸情况，对成絲强度往往有很大 关系，再生粘液䊹維的凝固条件对絲的結构及强度 也有密切关系。目前有相当多的研究工作者都在企 图进一步间明这些問題。

具有不同結构的程維，它們受热、氧化和受光 及輻射能的影响而产生結构的改变或裂解程度也是 不一致的。最近, 有些研究工作者正試图利用各种 新型工具(如气色譜、稹譜及順磁共振等) 来呼究这 些变化規律，以达到进一步对它进行控制的目的。

近来，在人造秆維及合成䊹維研究領域中，除 了上述的一些共同性基本問題外，还有很多新的研 究結果，現分別簡述如下:

人造释維: 目前在世界上产量最大的人造种維 还是粘液絲。然而它所存在的問題传然很多，首先 是如何广泛利用非木材糿維原料，用更适当的制浆 方法(如予水解法、溶剂法等)以制得粘液杍維的閒 題。目前，对多种䊹維原料能否适合作为化学紙浆 的要求及制定較滿意的測定其反应能力的方法問 題，还在継續研究中。前已进及，粘液釈維再生成 型过程中各种工艺条件对其結构和物性的影响均极 显著，因此，研究解决这些問題，是工业上制得高 强度粘液䊹維（如輪胎綫）必不可少的条件。近年 来, 为了进一步提高粘液絲的强度, 科学工作者研 究用长鍕媵类、醇类、聚乙烯等单独或混合加入粘 液及紡絲浴中，由于它們具有不同程度的保护胶体 的作用，能在一定程度上控制粘液再生的速度。樶 近，更有人研究用一些易裂解的水溶性双官能团的 正离子型化合物(包括含垟基、硫醇或胺类化合物) 使粘液再生成絲时产生少量交联，从而提高产品强 度，降低潤胀性能，取得了沓著的效果。但是，交 联分子的大小和交联数量的控制，与最終性能有着 极为密切的关系，因此，研究这类問題，无論在理 論上与实践上均有重大的意义。

除再生秆維如粘液絲外，糿維素經化学处理后 变为具有一些新性能的人造糿維, 虽然早已有硝酸 釈維及醋酸䊹維見諸于大規模生产，但塭近几年 来，改变納維原有結构与性能的弱点，使紒維素䊹 維变性，已有了愈来愈多的途径。抹且，这已成为 有关研究領域与工业生产发展中的一个新方向。磁 究已較成熟而已用于生产者，如棉紒維經局部乙醇 


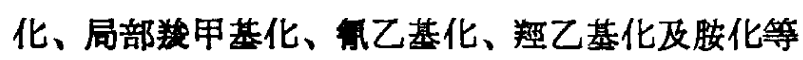
处理后，其程弹性、耐摩擦性、耐槢緆性、抗細 菌及抗化学获剂性能、染色活性、抗燃燒性能及湿 强度等，都有了个別的或全面的提高。

如上所述，利用一些易裂解的双官能团的正离 子化合物或其他活性多官能团化合物处理糿維素糿 㒕，使其大分子間形成一定数量的交联，且使其間 产生松弛处，那么这种新型秆維便会具有較高的耐 洗滌性及酎楔緭性，它比一般用合成树脂处理来㹨 得这些性能的方法有着本质上的优点。

利用一些活性烯类化合物（如烯醇胺、烯醇类 等）与杆䧴素生成醚类，預期可以得到具有較高的 染色或离子活性及参加化学反应活性的秆維。

为了使糿維染色活性及年度增加，一些研究工 作者正試驗用各种染料分子直接与秆維素化合，使 它成为化学键結合的糿維，不过其中有些途径距离 实用还較䓕。

改进糿維素的抗燃燒性能, 除了前述的 THPC、 APO 及 BAP 和維外，最近更有人研究用另一些有 机磷化合物及秄維的甲基及苯甲基磺醇化的衍生 物，再經澳化及碘化后，得到具有較高的耐洗涤性 能以及抗燃燒性能的䊹維。

具有高結晶度的天然䊹維素(麻、棉等)固然有 較高的强度，但在伸长、染色活性等方面还不能完 全令人㴖意。过去，曾有人研究过用碰液使其产生 物理結构的改变，邞早已付諸生产。近年来，用液 统及有机胺类处理麻棉秆維，使之脫晶及去取向的 工作还在継綂研究中。

最近以来，一个全新的具有很大发展前途的方 向，是用合成高分子与天然糿維素生成共聚物，使 原来維性能产生本稹的变化。这种变化，不只是 由于第二組成能引起化学結构的改变, 而且也能使 秄維超分子序态結构、大分子間松弛与紧密情况发 生差异。事实証明，从共聚高分子制得的秄維，在 性能上总是具有原来两种单一高聚物的个別优点, 有时还可以得到原来都没有的一些新性稹。因此, 有人把这种共聚物喻为高分子的合金。正如前面所 述，由于这类工作还仅处于萌芽阶段，因此，研究 达到共聚結构的适宜途径，共聚过程的基本 規律， 控制生成結构及測定結构的实际状态及其与物理化
学性能的关系，将为高分子化合物領域开辟既有生 产价值又有科学意义的重要方向。

从理論上預測，納維素与現有的合成高聚物似 乎都有生成共聚物的可能性，但是，到目前为止， 在研究工作中还只能得到接枝共聚物。而在接枝途 径上，也还不是所有縮聚与聚合等反应，均能完全 适合糿維素与其单体进行接枝共聚。目前，已有初 步結果的有:（1)納維素与甲基丙烯酸經游离基聚合 生成接枝共聚物;（2）秆維素或其衍生物与聚酸胺 型高聚物 (如尼龙 6 及 66 等) 生成接枝聚合物。目 前, 研究結果还未完善, 特別是对結构状态与物性 还不能作出明确的說明。但从很簡单的一些数据已 可看出，在物性方面有显著的变化。以糿維素与聚 丙脪腈接枝产物为例，其耐㱆性能就有 显 著的 提 高，远非納維素經氧乙基化的产物所能及; 其他如 伸度、染色活性及种維比重等，也都有不同程度的 改进。从共聚的途径来看, 近来采用的有: (1) 将 䊹䧴素或其行生物先加上适当的双軴, 再进行接枝 聚合;（2）选择适宜的引发剂，使䊹維大分子本身 产生游离基；（3）秆維素或其衍生物經臭氧化而产 生过化基团;（4）糿維素改变成含一定特性官能 团的高分子，再与适宜的合成高分子經交換反应而 接枝(如与尼龙 66 及蛋白的接枝等); (5) 䊹維素及 其衍生物經光及輻射能作用，而与其他烯类单体产 生接枝共聚物。㲤无疑問，愈来愈多的研究工作， 将集中于深入解决与此有关的一系列問題。

合成糿維方面: 近年来，关于高分子化合物的 聚合过程，其結构与性能之間的关系的研究让日益 深入，从更多新型聚合物中寻找合成維的工作也 正迅速发展着, 因此, 随之而来的研究与生产上的 問題也必然日经增加。

在縮聚反应方面，(一般聚酯及聚䤈胺等均属 这种反应类型)，目前除在探索更适宜的催化 剂及 工艺条件外，正通过一些新的聚合方法以改进原来 聚合方式的缺点, 例如正在开始扑逐濑哚入研究固 相聚合、界面聚合的基本規律及适用范围。与此同 时, 关于聚合物的裂构 (受热、光、氧化的影响)机 理及控制，結构与机械性能(首先是成絲可能性)方 面的有关研究，也在不断丰富中。此外，較多的研

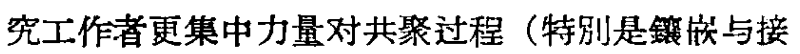


枝)进行研究以改进目前已有的这类糿維在性 能 上 的一些缺点（如染色活性低，溶解性不长，热稳定 性差等)，扶已初步获得了一些效果。但是，在对 共聚物結构的測定与控制及对其物性的明确方面的 研究，由于方法的局限，还是最薄弱的一环。因 此，䋱有大量的新型共聚物合成出来，但投入生产 者还不多。

在聚合反应方面，过去对游离基聚合反应的有 关因素及規律的研究虽較成熟，但对离子型聚合反 应結果的积界則較少。近年来，研究阳离子型聚合 以制得聚丙烯腈，其結果虽不能命人十分滿意，但为 了制备热稳定性更佳的維，这个途径还是比較理 想的。最近几年来，利用碱性催化剂通过阳离子型 聚合制备已队酭胺的硑究成果，已逐漸增多，大大 改变了通过縮聚反应制备已內酸胲时需要高溫及較 长时間的缺点。从一系列实驗証明，在阳离子型聚 合反应中，反应溫度可降到 $80-120^{\circ} \mathrm{C}$ ，而聚合时 間則可減少到 3-30 分鈡不等; 聚合体分子量可达 到抽絲要求，只是在分子量分布均一性上，还有待 継程研究控制的方法。另外，这些反应的机构及催 化剂的选择等等，都是具有重大意义的問題。目前 在离子型聚合中，发現在一些特殊的催化剂及一定 的反应条件下，可以得到具有规則的室間构向的聚 合物，咱定向結构的聚合物。它在物性方面，較同 一的但其有不规則空間构向的产品具有特殊的优异 之处，这从下表可見一二。

在乙烯及丙烯等聚合过程中，如果在高压下經 游离基聚合，則可得主鏈較短而枝鏈較多的綫型聚

表 2 不同方法制得的聚丙烯的物性比較

\begin{tabular}{|c|c|c|}
\hline 物性指标 & 定向聚合 $(60 \%$ 結晶度) & 低压法 \\
\hline 密 度 & $0.90-0.91$ & 0.94 \\
\hline 熔 点 ${ }^{\circ} \mathbf{F}$ & $330-340$ & $270-278$ \\
\hline 屈伏強度（平方英吋） & $5150-5720$ & 3140 \\
\hline 伸 长 \% & $100-600$ & 700 \\
\hline
\end{tabular}

合体，而且結晶趋向极小。由齐格来型催化剂（三 乙基鋁及氮化鈦)及齐格来一娜塔型催化剂制得的聚 合物，則具有一定的結晶傾向，而且取代基均位于 问一空間平面，郎所謂定向聚合体。从現有結果可 以看出，綫型聚合物的定向度，与所用催化剂的离 子晶体結构有着极为密切的关系，不同結构的催化 剂，或同一化学結构而催化剂的物理結构状态有区 別者，所得聚合物的定向度也有显著差別。目前以 齐格来一娜塔催化剂为中心，已研究出具有不少类 似功效的催化剂，以进一步明确它对空間定向生成 的作用。显然，这也是一个具有十分重要意义的发 展方向。現在，从定向聚乙烯、丙烯、丁烯、苯乙 烯单聚或共聚得到的产品，大部分还只应用于橡胶 及望料工业，但已可初步証明，定向聚丙烯維仍 有它特殊的机械性能，在一定的工业应用要 求上， 也占有重要的地位。前已指出，为了改变䋐性单一 聚合物在結构与物性上所呈現的弱点，科学工作者 近年来正集中研究一些二元或三元共聚物，以通过 适宜途径㢸得一定結构的共聚体，了解各种組成在 共聚反应中体現的規律，进一步控制产品結构及明 确結构的埧实状态。这些問題都是必須研究抹予以 解决的。

除了上迌聚合过程外，最近在利用光、輻射能 以产生游离基及活性离子，从而达到聚合目的的研 究工作，也在飞速地增加。这种聚合方法特別是对 一些高聚物的接枝共聚更为适当。当然，这类問題 的研究还只是开始，要使它过渡到有实践意义，首 先必須明确，光及輻射能对大分子結构可以同时引 起的变化一一裂构及交联作用，聚合条件与这些作 用的相互关系，以及如何防止聚合过程中随之而产 生的裂构作用等。另一个予期有意义的研究工作， 垖通过高速度的机械研槩或超声波振燙，以引起聚 合作用。各种合成单体或高聚物生成接枝或镬宸共 聚物的可能性，都是存在的，如何进一步使其具有 更多的現实意义，这正是有关科学工作者应影努力 的方向。 